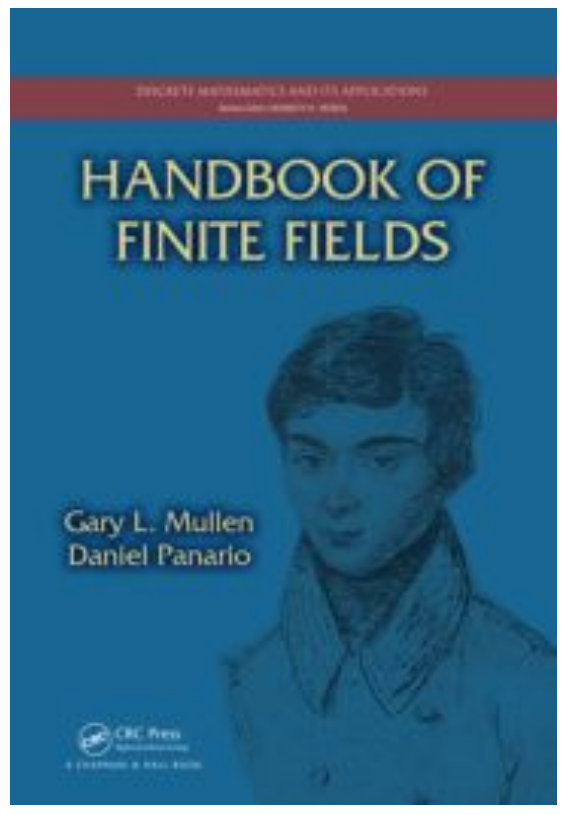

\title{
Handbook of Finite Fields
}

\author{
Hardback \$139.95 ISBN 9781439873786 \\ Cat\# K13417 \\ Series:
}

Discrete Mathematics and Its Applications

Published:

June 17, 2013 by Chapman and Hall/CRC

Content:

1068 Pages | 12 Illustrations

Author(s):

Gary L. Mullen; Daniel Panario

\section{Table of Contents}

\section{Introduction}

History of Finite Fields, Roderick Gow

Finite fields in the 18th and 19th centuries

\section{Introduction to Finite Fields}

Basic properties of finite fields, Gary L. Mullen and Daniel Panario

Tables, David Thomson

\section{Theoretical Properties}

\section{Irreducible Polynomials}

Counting irreducible polynomials, Joseph L. Yucas

Construction of irreducible, Melsik Kyuregyan

Conditions for reducible polynomials, Daniel Panario

Weights of irreducible polynomials, Omran Ahmadi

Prescribed coefficients, Stephen $D$. Cohen

Multivariate polynomials, Xiang-dong Hou

\section{Primitive Polynomials}

Introduction to primitive polynomials, Gary L. Mullen and Daniel Panario

Prescribed coefficients, Stephen D. Cohen 
Weights of primitive polynomials, Stephen D. Cohen

Elements of high order, José Felipe Voloch

\section{Bases}

Duality theory of bases, Dieter Jungnickel

Normal bases, Shuhong Gao and Qunying Liao

Complexity of normal bases, Shuhong Gao and David Thomson

Completely normal bases, Dirk Hachenberger

\section{Exponential and Character Sums}

Gauss, Jacobi, and Kloosterman sums, Ronald J. Evans

More general exponential and character sums, Antonio Rojas-León

Some applications of character sums, Alina Ostafe and Arne Winterhof

Sum-product theorems and applications, Moubariz Z. Garaev

\section{Equations over Finite Fields}

General forms, Daqing Wan

Quadratic forms, Robert Fitzgerald

Diagonal equations, Francis Castro and Ivelisse Rubio

\section{Permutation Polynomials}

One variable, Gary L. Mullen and Qiang Wang

Several variables, Rudolf Lidl and Gary L. Mullen

Value sets of polynomials, Gary L. Mullen and Michael E. Zieve

Exceptional polynomials, Michael E. Zieve

\section{Special Functions over Finite Fields}

Boolean functions, Claude Carlet

PN and APN functions, Pascale Charpin

Bent and related functions, Alexander Kholosha and Alexander Pott

$k$-polynomials and related algebraic objects, Robert Coulter

Planar functions and commutative semifields, Robert Coulter

Dickson polynomials, Qiang Wang and Joseph L. Yucas

Schur's conjecture and exceptional covers, Michael D. Fried

\section{Sequences over Finite Fields}

Finite field transforms, Gary McGuire

LFSR sequences and maximal period sequences, Harald Niederreiter

Correlation and autocorrelation of sequences, Tor Helleseth

Linear complexity of sequences and multisequences, Wilfried Meidl and Arne Winterhof

Algebraic dynamical systems over finite fields, Igor Shparlinski

\section{Algorithms}

Computational techniques, Christophe Doche

Univariate polynomial counting and algorithms, Daniel Panario

Algorithms for irreducibility testing and for constructing irreducible polynomials, Mark

Giesbrecht

Factorization of univariate polynomials, Joachim von zur Gathen

Factorization of multivariate polynomials, Erich Kaltofen and Grégoire Lecerf

Discrete logarithms over finite fields, Andrew Odlyzko

Standard models for finite fields, Bart de Smit and Hendrik Lenstra

\section{Curves over Finite Fields}

Introduction to function fields and curves, Arnaldo Garcia and Henning Stichtenoth

Elliptic curves, Joseph Silverman

Addition formulas for elliptic curves, Daniel J. Bernstein and Tanja Lange 
Hyperelliptic curves, Michael John Jacobson, Jr. and Renate Scheidler

Rational points on curves, Arnaldo Garcia and Henning Stichtenoth

Towers, Arnaldo Garcia and Henning Stichtenoth

Zeta functions and L-functions, Lei Fu

$p$-adic estimates of zeta functions and L-functions, Régis Blache

Computing the number of rational points and zeta functions, Daqing Wan

Miscellaneous Theoretical Topics

Relations between integers and polynomials over finite fields, Gove Effinger

Matrices over finite fields, Dieter Jungnickel

Classical groups over finite fields, Zhe-Xian Wan

Computational linear algebra over finite fields, Jean-Guillaume Dumas and Clément Pernet

Carlitz and Drinfeld modules, David Goss

\title{
Applications
}

\section{Combinatorial}

Latin squares, Gary L. Mullen

Lacunary polynomials over finite fields, Simeon Ball and Aart Blokhuis

Affine and projective planes, Gary Ebert and Leo Storme

Projective spaces, James W.P. Hirschfeld and Joseph A. Thas

Block designs, Charles J. Colbourn and Jeffrey H. Dinitz

Difference sets, Alexander Pott

Other combinatorial structures, Jeffrey H. Dinitz and Charles J. Colbourn

$(t, m, s)$-nets and $(t, s)$-sequences, Harald Niederreiter

Applications and weights of multiples of primitive and other polynomials, Brett Stevens

Ramanujan and expander graphs, M. Ram Murty and Sebastian M. Cioaba

\author{
Algebraic Coding Theory \\ Basic coding properties and bounds, Ian Blake and W. Cary Huffman \\ Algebraic-geometry codes, Harald Niederreiter \\ LDPC and Gallager codes over finite fields, Ian Blake and W. Cary Huffman \\ Turbo codes over finite fields, Oscar Takeshita \\ Raptor codes, Ian Blake and W. Cary Huffman \\ Polar codes, Simon Litsyn
}

\section{Cryptography}

Introduction to cryptography, Alfred Menezes

Stream and block ciphers, Guang Gong and Kishan Chand Gupta

Multivariate cryptographic systems, Jintai Ding

Elliptic curve cryptographic systems, Andreas Enge

Hyperelliptic curve cryptographic systems, Nicolas Thériault

Cryptosystems arising from Abelian varieties, Kumar Murty

Binary extension field arithmetic for hardware implementations, M. Anwarul Hasan and Haining Fan

\section{Miscellaneous Applications}

Finite fields in biology, Franziska Hinkelmann and Reinhard Laubenbacher

Finite fields in quantum information theory, Martin Roetteler and Arne Winterhof

Finite fields in engineering, Jonathan Jedwab and Kai-Uwe Schmidt

\section{Bibliography \\ Index}

